\title{
Técnica quirúrgica modificada en la Enfermedad de La Peyronie. Nuestra experiencia
}

\author{
Candebat Montero LH*, Barbosa Ramos F*, Rabionet Carballo F*, González Ferro I**, \\ Tamayo Tamyo I*, Giraudy Simón G*.
}

*Servicio de Urología. Hospital Provincial Clinico Quirúrgico Universitario Saturnino Lora Torres. **Servicio de Imagenologia. Hospital Clínico Quirúrgico Juan Bruno Zayas Alfonso. Santiago de Cuba. Cuba.

Actas Urol Esp. 2008;32(2):240-245

\section{RESUMEN}

\section{TÉCNICA QUIRÚRGICA MODIFICADA EN LA ENFERMEDAD DE LA PEYRONIE.} NUESTRA EXPERIENCIA

Introducción: La enfermedad de La Peyronie consiste en la formación de una o varias placas fibrosas, que además de afectar la túnica albugínea y alrededor del tejido cavernoso restringen no sólo la expansión del pene durante la tumescencia, sino la de ese segmento del conducto peneano, con lo cual generan una erección curva. Se conocen varias modalidades terapéuticas. El objetivo de este trabajo es proponer en esta patología una modificación a la técnica quirúrgica de Essed y precisar la evolución de estos pacientes.

Método: Se llevó a efecto un estudio descriptivo y transversal entre el 2003 y el 2005 con el propósito de modificar una técnica quirúrgica en sujetos con enfermedad de La Peyronie. Se incluyeron en esta investigación 210 pacientes con edades entre los 30 y 70 años con diagnóstico clínico y ecográfico de esta patología. Cada sujeto firmó previamente un documento (consentimiento informado de participación). Se midió la curvatura peneana previa cirugía y se valoró la función sexual según el índice internacional de función eréctil (IIFE).

Resultados: Predominó el grupo etáreo de 45 a 60 años con el $62 \%$ y aquellos pacientes con sintomas de más de un año de evolución para el 52,4\%. La hipertensión arterial, diabetes Mellitus y el síndrome Kelami se señalan como los antecedentes personales más frecuentes, con el 35,2; 19,7; y 7,2\% respectivamente $\mathrm{El}$ 98,6 \% de los pacientes tuvieron solución quirúrgica definitiva a los 6 meses post cirugía y el resto a los 9 meses.

Conclusiones: El examen ecográfico constituyó un medio eficaz para una mejor definición de la localización, cuantificación y tamaño de las placas. La modificación quirúrgica propuesta, evita las complicaciones de la operación original y tiene un extraordinario impacto económico al disminuir costos por ser una cirugía ambulatoria, con anestesia local y sin complicaciones

Palabras clave: Técnica de Essed. Enfermedad de La Peyronie.

\section{ABSTRACT}

MODIFIED SURGICAL TECHNIQUE IN PEYRONIE'S DISEASE. OUR EXPERIENCE.

The authors conducted a study from 2003 to 2005 with the purpose of modifing a surgical technique in subjects with Peyronie's disease. Each subject filled out and signed an informed consent form established for use by the Hospital Provincial Universitario "Saturnino Lora Torres", in Santiago de Cuba, that included a description of the variables of the investigation. The study confirmed that Peyronie's disease affecting predominatly males between the ages of 30 to 70 years. Dorso lateral incurvation was the most comon. The ecogram is an effective method for locating and measuring plaque.

Results: Total correction of the curvature in 98,6\%. High grade of satisfaction in 200 patients .

Conclusion: Essed operation is an easy and effective surgical technique in the Peyronie's disease for curvature correction. The proposed surgical technique avoided the complications found in original operations and has an economic impact of reducing costs by providing an ambulatory surgery using local anesthesic. 
$\mathrm{L}$ a enfermedad de La Peyronie, también conocida como la esclerosis fibrosa del pene o induración plástica del pene, aunque se conoce fue descrita en 1743 por Francois Gigot de La Peyronie (1678-1747), cirujano de Luis XIV, en un paciente "con una cicatriz en forma de cuentas en rosario que le causaba una curvatura del pene hacia arriba durante la erección", ya en 1561 Falopius escribe acerca de ella ${ }^{1-4}$.

El trauma del pene erecto, que a veces puede ser mínimo, pero que en individuos susceptibles provoca la lesión microvascular repetitiva, no desaparece como ocurre en la reparación normal, ya sea debido a una incapacidad para eliminarla o una deposición adicional de fibrina, consecutiva al reiterado traumatismo ${ }^{9}$.

Los pacientes sufren dolor a la erección peneana y pobre consistencia en la zona de curvatura. La deformidad del órgano puede ser severa e impedir una penetración vaginal satisfactoria, que influye en el estado psíquico de los afectados ${ }^{4-6}$.

En el examen físico del órgano, se observa un área fibrosa densa, de variado tamaño que se localiza en la albugínea de los cuerpos cavernosos, cerca de la línea del eje peneano. A veces existen varias placas, distribuida como sigue: 75 a $80 \%$ de ubicación dorsal, 20 a $25 \%$ lateral y 7 a un $9 \%$ ventral, según nuestra experiencia y datos internacionales ${ }^{5-9}$.

Se conocen varias modalidades terapéuticas, algunas de las cuales se refieren a mantenerse expectantes ante la aparición de dolor o curvatura mínima como único síntoma, pues se dice que su resolución puede ocurrir espontáneamente entre $15-50 \%$ de los casos. Otras recomiendan indicar un tratamiento medicamentoso, en la fase inflamatoria, basado en: Vitamina E, Interferón beta o alfa recombinante, corticoides, Ppropóleos (en cápsulas), Procarbazina, Potaba (o pabex o paraaminobenzoato de potasio), Orgoteina (u ontosein o superóxido dismutasa), Alopurinol, Colchicina y Verapamilo, entre otros. También se reporta el uso de Iontoforesis con la aplicación tópica de fármacos o infiltración de estos, así como láser terapia ${ }^{9-11}$.

Son numerosas las técnicas quirúrgicas ${ }^{12-15}$ empleadas para la corrección de la deformidad ocasionada por esta enfermedad. Para su mejor comprensión las clasificamos en:
A. Técnicas de plicatura o plastia de la túnica albugínea

- Su objetivo es el enderezamiento del pene, no se toca la fibrosis de la placa y se actúa sobre la convexidad del pene, rectificándola.

- Nesbit: Se resecan varias elipses de albugínea y sutura de ellas.

- Plicatura simple (Essed-Shroeder y Knispel) conocida como técnica de Essed: Se realizan varias plicaturas de la túnica albugínea.

- Variantes (Sassine, Licht, entre otras).

B. Técnicas de incisión - excisión de la placa: Devine y Horton.

Montorsi.

\section{OBJETIVOS}

1. Proponer en esta patología una modificación a la técnica quirúrgica de Essed.

2. Precisar la evolución de estos pacientes.

\section{MATERIAL Y MÉTODOS}

Caracteristicas generales de la investigación

Se llevó a efecto un estudio descriptivo y transversal con el propósito de modificar una técnica quirúrgica en sujetos con enfermedad de La Peyronie y precisar la evolución de estos pacientes. A cada paciente se le aplicó un formulario (Anexo), que contenía las variables, en consulta externa de Urología habilitada para tales fines en el Hospital Provincial Universitario "Saturnino Lora Torres", de Santiago de Cuba.

A doscientos diez pacientes con curvatura adquirida del pene a la erección se les realizó la modificación a la técnica quirúrgica de SchroederEssed, desde el 2003 hasta el 2005.

\section{Técnicas y procedimientos}

Para obtener la información necesaria se llevó a cabo un examen físico del pene, donde se palpó la(s) placa(s) fibrosa(s) al nivel de los cuerpos cavernosos y posteriormente se utilizó un instrumento milimetrado para medir el tamaño de ésta(s) placa(s), siempre por el autor del trabajo. Luego se realizó una ecografia diagnóstica del pene (con escala de grises en modo B) a cada paciente con un equipo SIEMENS de 7,5 megahercios.

Se efectuaron cortes coronales y sagitales en el pene para definir el tamaño de las placas y su localización en cada cuerpo cavernoso. 
Anexo. Formulario o encuesta

Nombres y apellidos

Dirección particular

Historia clínica

1. Edad (en años)
a) $30 \mathrm{a}<45$
b) $45 a<60$
( )
c) 60 y más

2. Antecedentes patológicos personales

a) Hipertensión arterial

b) Diabetes Mellitus

c) Enfermedades del colágeno

d) Trastornos vasculares

e) Otros

4. Dirección de la incurvación del pene a la erección
a) Hacia arriba
b) Hacia abajo
( )
c) Lateral

5. Dolor a la erección del pene
a) $\mathrm{Si}$
b) $\mathrm{No}$

6. Dificultad para las relaciones sexuales

a) $\mathrm{Si}$

b) $\mathrm{No}$

7. Número de placas al examen físico
a) Únicas
b) Múltiples
c) Ausentes

8. Localización de las placas fibrosas al examen físico
a) Dorsal
$($ )
b) Ventral
( )
c) Lateral

9. Tamaño de las placas fibrosas al examen físico
a) Menor de $3 \mathrm{~mm}$
b) $3 \mathrm{~mm}$ y más

10. Número de placas mediante la ecografía
a) Únicas
b) Múltiples
c) Ausentes

11. Localización de las placas fibrosas mediante la ecografia
a) Dorsal
b) Ventral
c) Lateral

12. Tamaño de las placas fibrosas mediante la ecografía
a) Menor de $3 \mathrm{~mm}$
b) $3 \mathrm{~mm}$ y mas

Este procedimiento complementario fue realizado siempre por la misma especialista y con el mismo equipo de radiografía, para evitar los errores imputables al observador.

La operación original fue descrita por SchroederEssed en 1982 y consiste en una plicatura simple de la albugínea, que tiene como complicaciones quirúrgicas más importantes: la cortedad del pene de 1 a $2 \mathrm{~cm}$, la sepsis de herida quirúrgica, el hematoma peneano y la palpación de los puntos de plicatura.

La modificación a esta técnica consistió:

1. Realizar la circuncisión a todos los pacientes.

2. Efectuar dos incisiones paralelas longitudinales de aproximadamente $5 \mathrm{~mm}$ a todo lo largo de la túnica albuginea en el sitio de la curvatura.

3. Realizar varias suturas continuas de plicatura, en vez de una sola discontinua, con la finalidad de evitar el acortamiento del pene.

4. Aplicar puntos invertidos para evitar que se palpen las suturas en el post-operatorio.

5. Utilizar anestesia local y ser en régimen ambulatorio.

\section{TÉCNICA QUIRÚRGICA}

1. Circuncisión en todos los pacientes.

2. Disección de la piel hasta la base del pene, con exposición de la fascia de Buck (Fig. 1).

3. Ligadura de la base del pene con un torniquete.

4. Puncionamos los cuerpos cavernosos con Scalp vein set (23 G) o con una aguja 19 G (Fig. 2).

5. Instilamos 100 cc de Cloruro de Sodio al 0,9\% con 1 bulbo de una Cefalosporina de tercera generación, hasta lograr la erección artificial.

6. Visualizamos el sentido de la curvatura peneana y marcamos los puntos donde realizaremos las suturas (Fig. 3).

7. Retiramos la ligadura de la base de pene.

8. Procedemos a efectuar dos incisiones paralelas longitudinales de aproximadamente 5 $\mathrm{mm}$ a todo lo largo de la túnica albugínea en el sitio de la curvatura (Fig. 4). 
9. Suturas múltiples, invertidas, hasta lograr la corrección de la desviación.

10. Utilizamos Polyester o Acido Poliglicólico 2/0. 11. Ligadura de la base peneana.

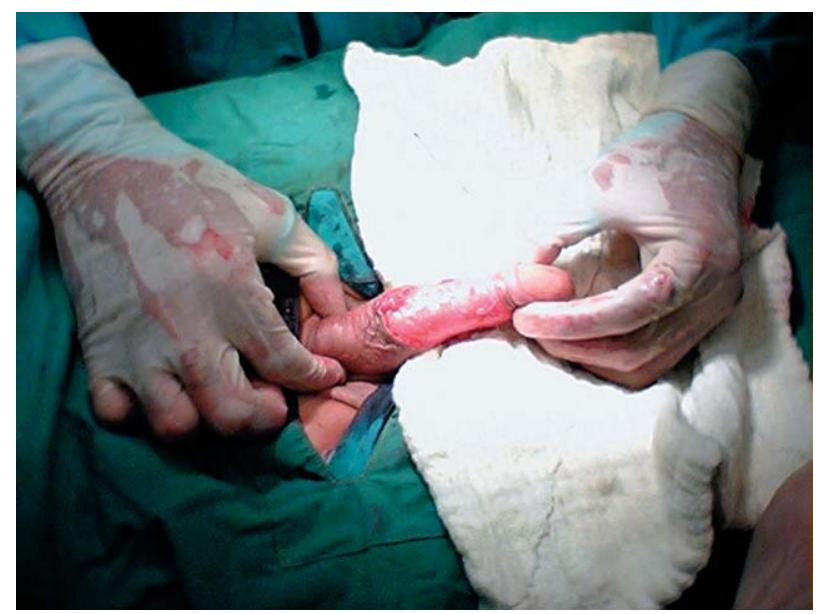

FIGURA 1

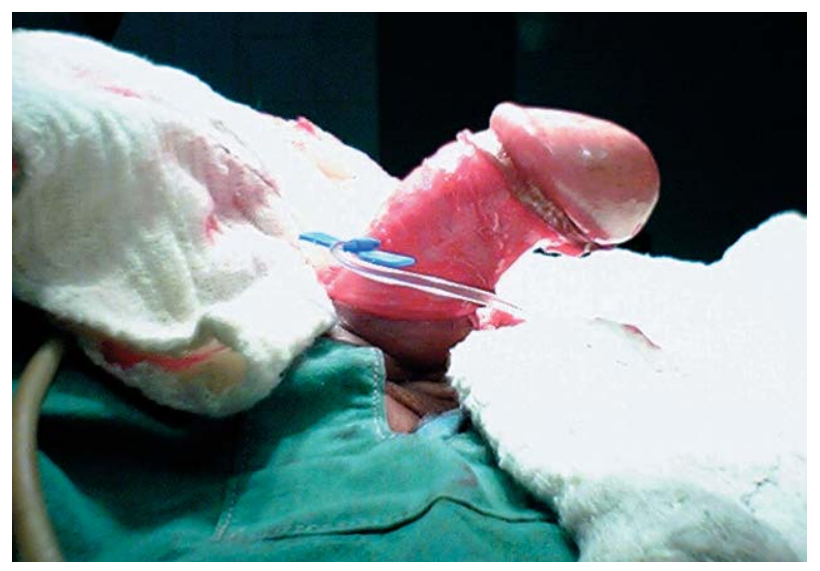

FIGURA 2

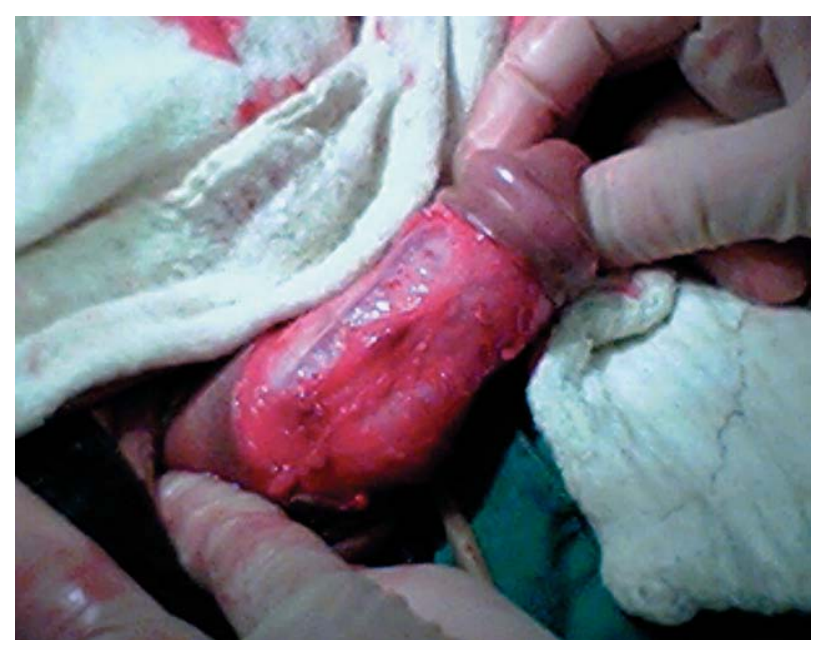

FIGURA 3

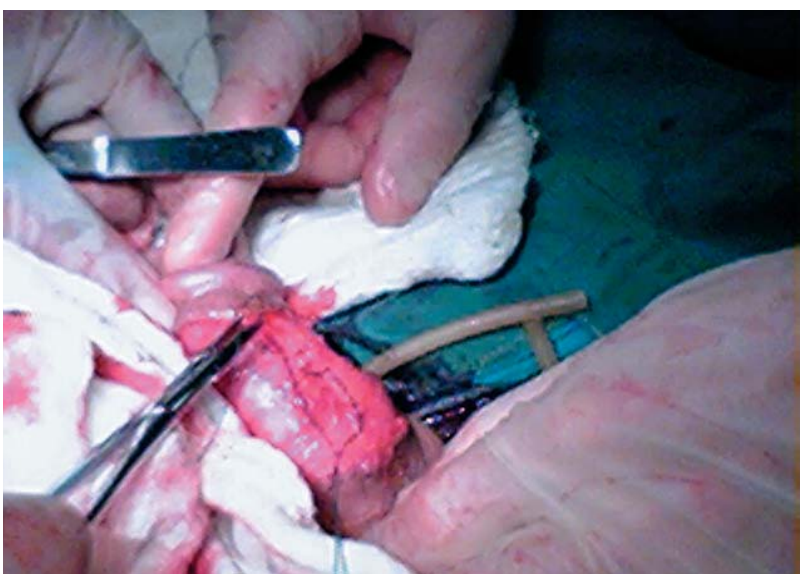

FIGURA 4

12. Confirmamos que el pene ha sido enderezado (Fig. 5 y 6)

13. Cierre por planos con Catgut Crómico $3 / 0$ incluyendo la sutura de la piel.

14. Vendaje compresivo del pene por 24 horas. Resultado final (Fig. 7 y 8).

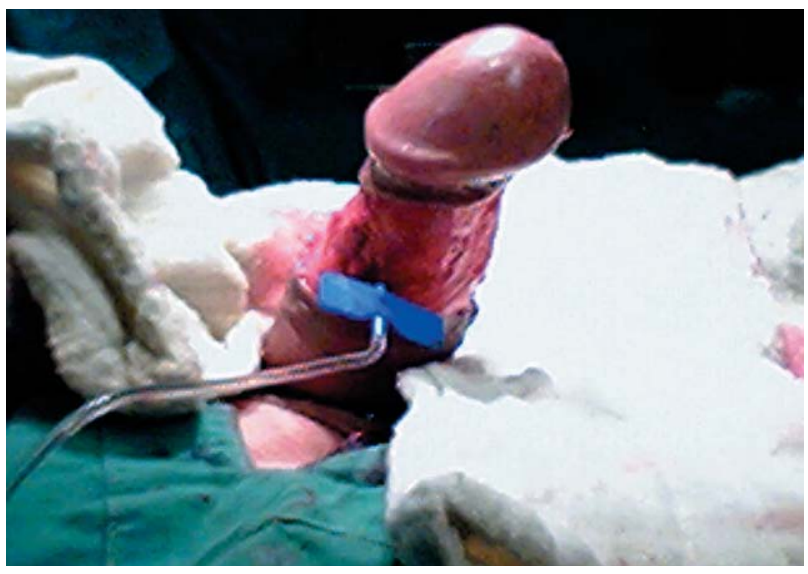

\section{FIGURA 5}

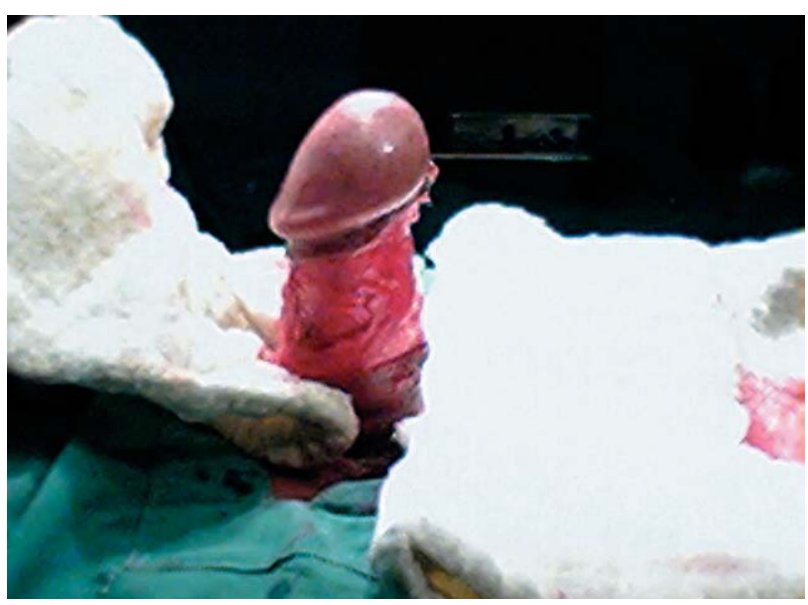

FIGURA 6 


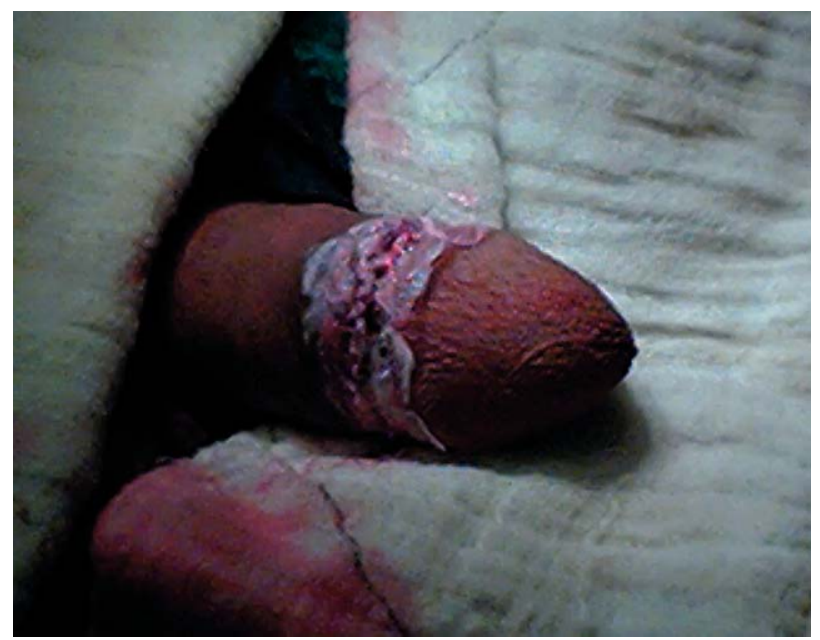

FIGURA 7

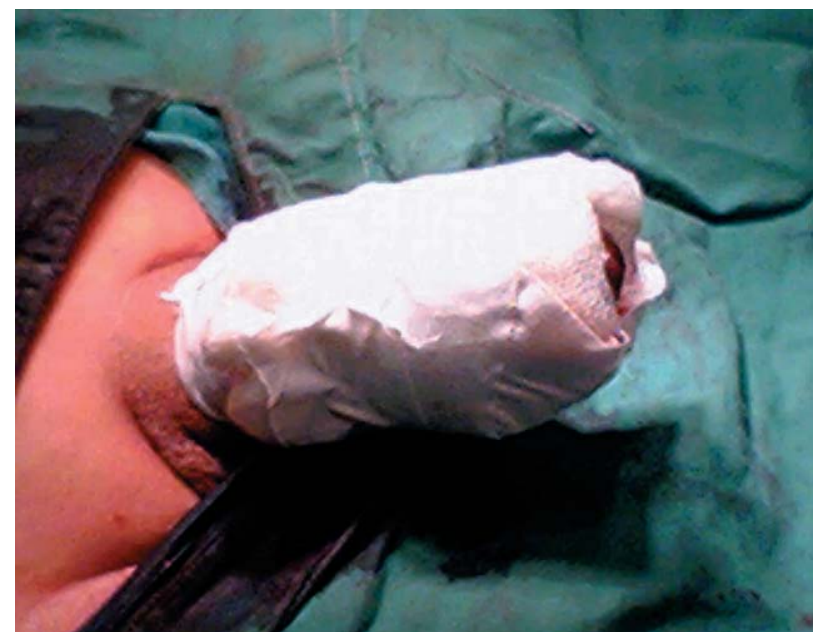

FIGURA 8

\section{RESULTADOS Y DISCUSIÓN}

En la Tabla 1, donde se analiza la edad de los pacientes en relación con el tiempo de aparición de los sintomas, observe cómo predominó el grupo etareo de 45 a 60 años con (62\%) seguido en orden por los mayores de 60 (24\%), y como sobresalió además, la aparición de los síntomas durante más de un año el $52,8 \%$ de la casuística, lo cual es estadísticamente significativo y se corresponde con lo registrado en la literatura extranjera ${ }^{4,5,9}$.

También se pudo comprobar que a mayor edad (45 años en adelante) los pacientes demoraron más en asistir a consulta externa, a pesar de ser la etapa de la vida en la que suele prevalecer este trastorno, sin que fuera posible establecer la causa de esta conducta.

La distribución de pacientes según la dirección de la incurvación peneana a la erección se refleja en la Tabla 2. Note que el $61 \%$ de la serie la dirección era lateral (derecha o izquierda, indistintamente), en sólo 4,7\% hacia arriba y en el resto hacia abajo. Esto difiere de lo afirmado en la bibliografia consultada, donde prepondera la incurvación hacia arriba. Se sugiere realizar otros estudios para analizar el efecto del tamaño de la placa fibrosa, en cada localización, con respecto a la incurvación.

Tabla 2. Pacientes según dirección de la incurvacion peneana

\begin{tabular}{lcc}
\hline Dirección de la incurvacion & No. & \% \\
\hline Hacia Arriba & 10 & 4,7 \\
Hacia Abajo & 72 & 34,3 \\
Lateral & 128 & 61 \\
Total & 210 & 100,0 \\
\hline
\end{tabular}

Fuente: $(*)$

De los 100 enfermos que tenían una placa mayor de $3 \mathrm{~mm}$ según el estudio ecografico (Tabla 3); 52,4\% experimentaron las manifestaciones clínicas por más de un año.

Esto corrobora la importancia de acudir a consulta externa de Urología tan pronto como aparezcan los primeros síntomas de la enfermedad,

Tabla 1. Pacientes según grupos etáreos y tiempo de aparición de los sintomas

\begin{tabular}{|c|c|c|c|c|c|c|}
\hline \multirow[t]{3}{*}{ Edad (en años) } & \multicolumn{6}{|c|}{ Tiempo de aparición de sintomas (en meses) } \\
\hline & \multicolumn{2}{|c|}{ Menor de 12} & \multicolumn{2}{|c|}{12 y más } & \multicolumn{2}{|c|}{ Total } \\
\hline & No & $\%$ & No & $\%$ & No & $\%$ \\
\hline $30<45$ & 10 & 4,8 & 20 & 9,5 & 30 & 14,2 \\
\hline $45<60$ & 50 & 24 & 80 & 38 & 130 & 62 \\
\hline 60 y más & 40 & 19 & 10 & 4,8 & 50 & 23,8 \\
\hline Total & 100 & 47,6 & 110 & 52,4 & 210 & 100,0 \\
\hline
\end{tabular}

Fuente: Formulario $\left(^{*}\right)$

$\mathrm{P}<0,01$ 
Tabla 3. Pacientes según grupos etáreos y tiempo de aparición de los sintomas

\begin{tabular}{|c|c|c|c|c|c|c|}
\hline \multirow[t]{3}{*}{ Tamaño de placa } & \multicolumn{6}{|c|}{ Tiempo de aparición de síntomas (en meses) } \\
\hline & \multicolumn{2}{|c|}{ Menor de 12} & \multicolumn{2}{|c|}{12 y más } & \multicolumn{2}{|c|}{ Total } \\
\hline & No & $\%$ & No & $\%$ & No & $\%$ \\
\hline Menor de $3 \mathrm{~mm}$ & 90 & 42,8 & 20 & 9,6 & 110 & 52,4 \\
\hline 3 mm y más & 10 & 4,8 & 90 & 42,8 & 100 & 47,6 \\
\hline Total & 100 & 47,6 & 110 & 52,4 & 210 & 100,0 \\
\hline
\end{tabular}

Fuente: $\left(^{*}\right)$

$\mathrm{P}<0,01$

así como realizar otras investigaciones en nuestra población para identificar los factores de riesgo y poder actuar sobre ellos

La desviación peneana fue abolida en casi la totalidad de estos pacientes (Tabla 4). Esta técnica quirúrgica se realizó aplicando anestesia local infiltrativa, en régimen ambulatorio y sin complicaciones manifiestas.

Tabla 4. Porcientos de resolución postcirugía

\begin{tabular}{cc}
\hline Según desviación peneana & \\
Lateral & 98,5 \\
Hacia arriba & 90,2 \\
Hacia abajo & 95,7 \\
\hline
\end{tabular}

El seguimiento después de la cirugía fue trimestral hasta el año y posteriormente anual por dos años.

E1 98,6 \% de los pacientes tuvieron solución quirúrgica definitiva a los 6 meses post cirugía y el resto a los 9 meses.

\section{CONCLUSIONES}

1. La enfermedad de Peyronie tiene una evolución de carácter crónico, es muy frecuente en nuestro medio predomina entre la cuarta y sexta décadas de la vida y no existe uniformidad de criterios en su terapéutica.

2. Esta modificación a la técnica quirúrgica de Essed, permite eliminar las complicaciones de la técnica original y reduce costos por concepto de evitar ingresos, haber utilizado anestesia local y reincorporarse rápidamente a la sociedad estos pacientes.

\section{REFERENCIAS}

1. Peyronie F. Sur quelques obstacles qui s' opposent a' 1' yaculation naturelle de la semence. Mem Acad Chir. 1743; 1:138.

2. Gingell JC, Desai KM. Peyronie's disease. Brit J Urol. 1989; 63(3):223-226.

3. Sarramon JP, Ecourrou G. The diagnosis and management of Peyrone's disease. Int J Impotence Res. 1991;3:69-72.

4. Gelbard MK, Dorey F, James K. The natural history of Peyronie's disease. J Urol. 1990;144(6):1376-1379.

5. Devine, CJ. International conference of Peyronie's disease. Introduction, J Urol. 1997;157(1):272-277.

6. Gingell JC, Desai KM. Peyronie's disease. Br J Urol. 1989; 63(3):223-226.

7. Somers KD, Dawson DM. Fibrin deposition in Peyronie's disease plaque. J Urol. 1997;157(1):311-315.

8. Rompell R, Muerller Eckhardt G, Schroeder- Printzen J, Weidner IV. HLA antigens in Peyronie's disease. Urol Int. 1994;52:134-9.

9. Devine CJ, Somers KD, Jordan GH, Schlossberg SM.: Proposal: trauma as the cause of the Peyronie's lesion. J Urol. 1997;157:6.

10. Wegner HE. Treatment of Peyronie's disease with local interferon-alpha 2b Evr. Urology. 1995;236-40-57.

11. Lobato R. Tratamiento de la enfermedad de Peyronie con interferón beta natural. Bol Col Mex Urol. 1997;14:28.

12. Levine, LA. International Verapamil injection for the treatment of Peyronie's disease. J Urol. 1994;1522:4-5.

13. Melfte AR. Corporeal plication for surgical correction of Peyronie's disease. Urology, 1990;144:281.

14. Claes H, Baert L. Corporeal plications for surgical correction in Peyronie's disease improve rigidity. Int J Import Res. 1995;119: 22.

15. Ralph DJ. The Nesbit operation for Peyronie's disease: 16 -years experience. J Urol. 1995;1362:3.

Correspondencia autor: Dr. L.H. Candebat Montero Servicio de Urología.

Hospital Provincial Clínico Quirúrgico Universitario Saturnino Lora Torres. Santiago de Cuba. Cuba Tel.: 5322641003

E-mail autor: 1hcandebat@yahoo.es

Información artículo: Original - Andrología

Trabajo recibido: julio 2007

Trabajo aceptado: octubre 2007 